\title{
Activity of and Resistance to Moxifloxacin in Staphylococcus aureus
}

\author{
Dilek Ince, Xiamei Zhang, and David C. Hooper* \\ Division of Infectious Diseases and Medical Services, Massachusetts General Hospital, \\ Harvard Medical School, Boston, Massachusetts 02114-2696
}

Received 17 September 2002/Returned for modification 25 November 2002/Accepted 20 December 2002

\begin{abstract}
Moxifloxacin has enhanced potency against Staphylococcus aureus, lower propensity to select for resistant mutants, and higher bactericidal activity against highly resistant strains than ciprofloxacin. Despite similar activity against purified $S$. aureus topoisomerase IV and DNA gyrase, it selects for topoisomerase IV mutants, making topoisomerase IV the preferred target in vivo.
\end{abstract}

8-Methoxyquinolones have been associated with increased bacteriostatic and bactericidal efficacy and the prevention of emergence of mutant strains in several bacteria, including Staphylococcus aureus $(4,5,12,23,33)$. Moxifloxacin is a new 8-methoxyquinolone with enhanced activity against gram-positive cocci, including ciprofloxacin-resistant $S$. aureus, with MICs 4- to 64-fold less than that of ciprofloxacin $(2,9,15,16$, $21,22,30,31)$. Although single mutations in topoisomerase IV also contribute to resistance, moxifloxacin preferentially selects for gyrase mutants in Streptococcus pneumoniae $(10,14)$, suggesting dual targeting of DNA gyrase and topoisomerase IV in $S$. pneumoniae $(29,32)$. In this study, we undertook to evaluate the primary target of moxifloxacin by selection of resistant mutants as well as inhibition of the DNA supercoiling activity of DNA gyrase and the decatenation activity of topoisomerase IV. The list of strains and plasmids used in the study are shown in Table 1.

Activity of moxifloxacin against genetically defined mutants of $S$. aureus. MICs of moxifloxacin and ciprofloxacin against genetically defined mutants of $S$. aureus determined by agar dilution on trypticase soy agar supplemented with twofoldincreasing concentrations of the antibiotics are shown in Table 2. Moxifloxacin was four- to eightfold more active against wildtype $S$. aureus ISP794 than ciprofloxacin. A single gyr $A$ mutation caused at most a twofold increase in the MICs of each quinolone, whereas a single mutation in either subunit of topoisomerase IV caused a four- to eightfold increase in MICs, suggesting topoisomerase IV to be the primary target of moxifloxacin in S. aureus. A double mutation in gyrase and topoisomerase IV caused a 125 -fold increase in the MICs of both quinolones, carrying the MIC of moxifloxacin to the proposed breakpoint of resistance of $\geq 4 \mu \mathrm{g} / \mathrm{ml}$ for staphylococci (1), although it remained below the achievable peak serum drug level of $4.73 \mu \mathrm{g} / \mathrm{ml}$ with an 800-mg dose of moxifloxacin (25). Thus, although moxifloxacin is more active than ciprofloxacin against mutants of $S$. aureus, it probably will not be effective against established strains with multiple resistance mutations. Overexpression of the NorA efflux pump caused at most a twofold increase in the MIC of moxifloxacin, consistent with

\footnotetext{
* Corresponding author. Mailing address: Division of Infectious Diseases, Massachusetts General Hospital, 55 Fruit St., Boston, MA 02114-2696. Phone: (617) 726-3812. Fax: (617) 726-7416. E-mail: dhooper@partners.org.
}

previously published results (20), probably due to the hydrophobicity of the 8-methoxy group as well as the bulkiness of the C-7 group (19, 27).

Bactericidal effects of moxifloxacin and ciprofloxacin against $S$. aureus. The bactericidal effects of moxifloxacin and ciprofloxacin at 4 times their respective MICs were determined by performance of time-kill curves as described previously against a wild-type $S$. aureus strain, ISP794, and its $\operatorname{grl} A$ and $\operatorname{grl} A$ gyr $A$ mutants (18). Colony counts were determined immediately before the addition of the antibiotics and hourly for $5 \mathrm{~h}$. Kill curves with each drug at 4 times the MIC showed similar effects for both the wild-type strain (Fig. 1A) and grlA mutant (Fig. 1B). The killing effect of ciprofloxacin appeared more pronounced (1-log difference) for ISP794 at the drug concentrations used $(1.0 \mu \mathrm{g} / \mathrm{ml}$ for ciprofloxacin and $0.128 \mu \mathrm{g} / \mathrm{ml}$ for moxifloxacin), perhaps due to the internal variation of MIC results (Fig. 1A). However, a 3-log decrease in colony counts for the grlA gyrA mutant was observed with moxifloxacin, whereas ciprofloxacin produced no killing (Fig. 1C). The concentration used for this double mutant $(16 \mu \mathrm{g} / \mathrm{ml})$, however, was above the achievable serum drug concentrations, and at simulated peak concentrations $(3 \mu \mathrm{g} / \mathrm{ml})$ moxifloxacin has been reported to lack bactericidal activity against highly resistant $S$. aureus isolates (6).

Frequency of selection of resistant mutants. Frequencies of a single-step mutation to resistance were determined by plating $S$. aureus ISP794 cells on brain heart infusion agar containing either moxifloxacin or ciprofloxacin at concentrations of 2,4 , and 8 times the MIC. The experiment was repeated three times, with plating of a maximum of $4.5 \times 10^{11} \mathrm{CFU}$. Mutation frequencies were calculated as the ratio of the number of resistant colonies at $48 \mathrm{~h}$ to the number of CFU inoculated. At 2 times the MIC of each quinolone, frequencies of selection of resistant mutants were similar (Table 3). However, at 4 times the MIC, mutants could rarely be selected with moxifloxacin, and the frequency of selection of resistant mutants was 3 orders of magnitude less than that of ciprofloxacin at 4 times the MIC. Mutants could not be selected at higher concentrations of moxifloxacin.

Characterization of single-step mutants. The MICs of moxifloxacin and ciprofloxacin increased two- to eightfold for three of the four single-step mutants studied (Table 2). MICs of nalidixic acid (used to screen for gyr $A$ mutations), novobiocin (used to screen for selected $\operatorname{grl} B$ or $\operatorname{grl} A$ mutations [8]), and ethidium bromide (used to screen for NorA overexpression [17]) 
TABLE 1. Bacterial strains and plasmids used in this study

\begin{tabular}{|c|c|c|}
\hline Strain or plasmid & Genotype $^{a}$ & Source or reference \\
\hline \multicolumn{3}{|l|}{ S. aureus strains } \\
\hline ISP794 & 8325 pig-131 & 24 \\
\hline MT5 & 8325 nov (gyrB142) hisG15 pig-131 & 7,28 \\
\hline SS1 & 8325 pig-131 gyrB142 (Ile102Ser, Arg144Lys) gyrA (Ser84Leu) & 3 \\
\hline MT5224c2 & 8325 nov (gyrB142) hisG15 pig-131 grlA552 & 18 \\
\hline MT5224c4 & 8325 nov (gyrB142) hisG15 pig-131 grlA542 (Ser80Phe) & 28 \\
\hline MT5224c9 & 8325 nov (gyrB142) hisG15 pig-131 grlB543 (Asn470Asp) & 28 \\
\hline MT23142 & 8325 pig-131 flqB $\Omega(\operatorname{chr}:: \operatorname{Tn} 916) 1108$ & 18 \\
\hline EN1252a & 8325 nov (gyrB142) hisG15 pig-131 grlA542 gyrA $\Omega 1051$ (Erm) $\mathrm{Nov}^{+}$ & 3 \\
\hline M2 & ISP794 grlA559 (Ala176Gly) & This study; spontaneous Mox ${ }^{\mathrm{r}}$ \\
\hline M3 & ISP794 (unidentified mutation) & This study; spontaneous $\operatorname{Mox}^{r}$ \\
\hline M4 & ISP794 grlA570 (Arg393Ser) & This study; spontaneous Mox ${ }^{\mathrm{r}}$ \\
\hline M6 & ISP794 grlA571 (Glu194Gly) & This study; spontaneous Mox $^{r}$ \\
\hline M8 & ISP794 grlA (Arg17His) (Glu87Lys) gyrA (Ser84Leu) & This study; serial passage mutant \\
\hline M11 & ISP794 gyrA (Ser84Leu), additional unidentified mutations & This study; serial passage mutant \\
\hline \multicolumn{3}{|l|}{ E. coli strains } \\
\hline $\mathrm{DH} 5 \alpha$ & 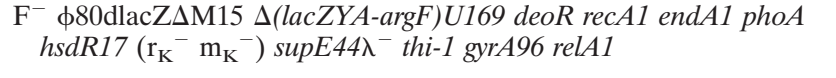 & GIBCO-BRL \\
\hline \multicolumn{3}{|l|}{ Plasmid } \\
\hline pTrcHisA, -B, and -C & 4.4-kb expression vector, $\mathrm{Ap}^{\mathrm{r}}$ & Invitrogen \\
\hline
\end{tabular}

${ }^{a}$ Abbreviations: $\mathrm{Mox}^{\mathrm{r}}$, moxifloxacin resistance; $\mathrm{Ap}^{\mathrm{r}}$, ampicillin resistance.

showed at most a twofold change (data not shown). The similar increase in the MICs of moxifloxacin and ciprofloxacin suggested that a mutation in topoisomerase IV was responsible for resistance, and direct sequencing of PCR products for the entirety of $\operatorname{grlBA}$ and the quinolone resistance-determining regions (QRDRs) of gyrBA performed using automated ABI 3100 DNA sequencers (Tufts Core Facility, Boston, Mass.) revealed two novel mutations outside the QRDR of $\mathrm{grlB}$ (Glu194Gly and Arg393Ser). Although genetic experiments were not performed to prove the role of these mutations in resistance, the absence of any other mutation in the entirety of $\operatorname{grlB} A$ suggested that these mutations were responsible for the resistance phenotype. The third mutant had a mutation in $\operatorname{grl} A$ (Ala176Gly), which we have previously selected with gatifloxacin (12). We have also previously, by allelic exchange experiments, demonstrated that another amino acid substitution at the same position in GrlA (Ala176Thr) is responsible for resistance (11). A fourth single-step mutant (M3) with a two- to fourfold increase in the MIC of moxifloxacin and at most a twofold increase in that of ciprofloxacin did not have any mutation in the entirety of $g r l B A$ and $g y r B A$. The MIC of ethidium bromide was also not increased for this strain, suggesting no increase in the expression of the efflux pump NorA. Se-

TABLE 2. Activity of moxifloxacin and ciprofloxacin against $S$. aureus

\begin{tabular}{|c|c|c|c|}
\hline \multirow{2}{*}{ Strain } & \multirow{2}{*}{ Mutation $^{a}$} & \multicolumn{2}{|c|}{$\operatorname{MIC}(\mu \mathrm{g} / \mathrm{ml})$} \\
\hline & & Moxifloxacin & Ciprofloxacin \\
\hline \multicolumn{4}{|c|}{ Genetically defined mutants of $S$. aureus } \\
\hline ISP794 & Wild type (parent) & 0.032 & $0.125-0.25$ \\
\hline MT5 & gyrB142 (Ile102Ser, Arg144Ile) & 0.064 & $0.25-0.5$ \\
\hline SS1 & gyrA (Ser84Leu) & 0.064 & 0.25 \\
\hline MT5224c2 & grlA (Ala116Pro) & $0.125-0.25$ & 1.0 \\
\hline MT5224c4 & grlA (Ser80Phe) & $0.125-0.25$ & 1.0 \\
\hline MT5224c9 & grlB (Asn470Asp) & $0.125-0.25$ & 1.0 \\
\hline MT23142 & $f l q B$ (NorA overexpression) & 0.064 & $0.5-1.0$ \\
\hline EN1252a & grlA (Ser80Phe) gyrA (Ser84Leu) & 4.0 & $16-32$ \\
\hline \multicolumn{4}{|c|}{ Single-step mutants selected in this study } \\
\hline M2 & grlA (Ala176Gly) & 0.25 & 1.0 \\
\hline M3 & Unidentified mutation & 0.125 & 0.25 \\
\hline M4 & grlB (Arg393Ser) & 0.25 & 1.0 \\
\hline M6 & grlB (Glu194Gly) & $0.125-0.25$ & 0.5 \\
\hline \multicolumn{4}{|c|}{ Serial-passage mutants selected in this study } \\
\hline M8 & grlA (Arg17His, Glu87Lys) gyrA (Ser84Leu) & 1.0 & 2.0 \\
\hline M11 & gyrA (Ser84Leu) and unidentified mutation(s) & 4.0 & 32 \\
\hline
\end{tabular}

${ }^{a}$ The indicated mutations in $g r l A$ in single-step or serial-passage mutants resulted from the following nucleotide changes: Arg17His, CGC $\rightarrow$ CAC; Glu84Lys, GAA $\rightarrow$ AAA, Ala176Gly, GCG $\rightarrow$ GGG. The indicated mutations in grlB or gyrB in single-step or serial-passage mutants resulted from the following nucleotide changes: Glu194Gly in $g r l B$, GAG $\rightarrow$ GGG Arg393Ser in $g r l B, C G T \rightarrow$ AGT, and Ser84Leu in gyrA, TCA $\rightarrow$ TTA. 

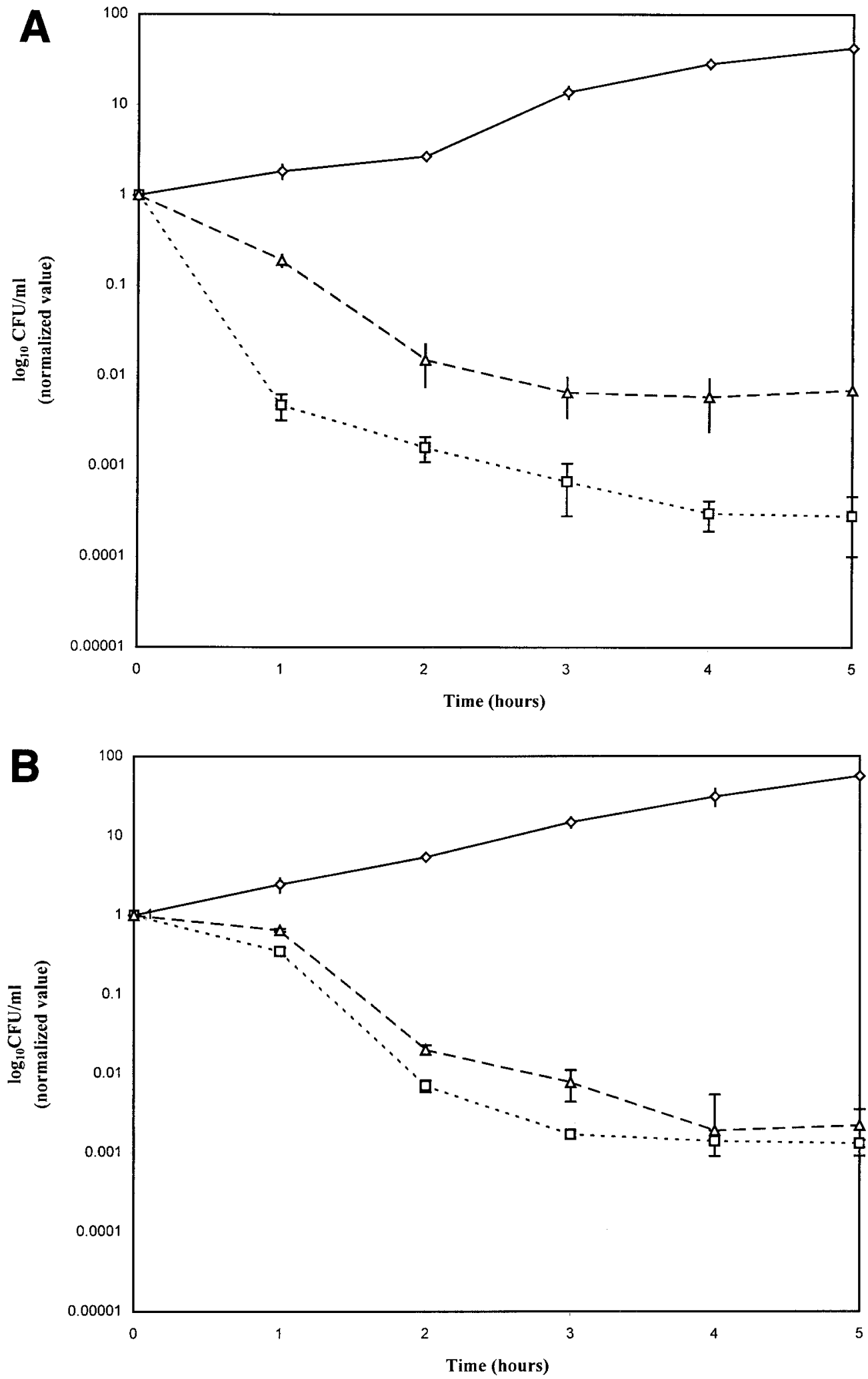

FIG. 1. Bactericidal activities of moxifloxacin and ciprofloxacin. Normalized viable counts of $S$. aureus strains in the presence of the indicated concentrations of moxifloxacin $(\triangle)$ and ciprofloxacin $(\square)$ or with no drug $(\diamond)$ are shown. (A) ISP794, wild-type $S$. aureus $\left(g r l A^{+}\right.$gyr $A^{+}$), at a ciprofloxacin concentration of $1.0 \mu \mathrm{g} / \mathrm{ml}$ and moxifloxacin concentration of $0.128 \mu \mathrm{g} / \mathrm{ml}$; (B) MT5224c4, grlA (Ser80Phe), at a ciprofloxacin concentration of $4.0 \mu \mathrm{g} / \mathrm{ml}$ and moxifloxacin concentration of $0.5 \mu \mathrm{g} / \mathrm{ml}$; (C) EN1252a, grlA (Ser80Phe) gyrA (Ser84Leu), at a ciprofloxacin concentration of $64 \mu \mathrm{g} / \mathrm{ml}$ and moxifloxacin concentration of $16 \mu \mathrm{g} / \mathrm{ml}$. $\diamond$, no antibiotic; $\triangle, 4$ times the MIC of moxifloxacin; $\square$, 4 times the MIC of ciprofloxaci. 


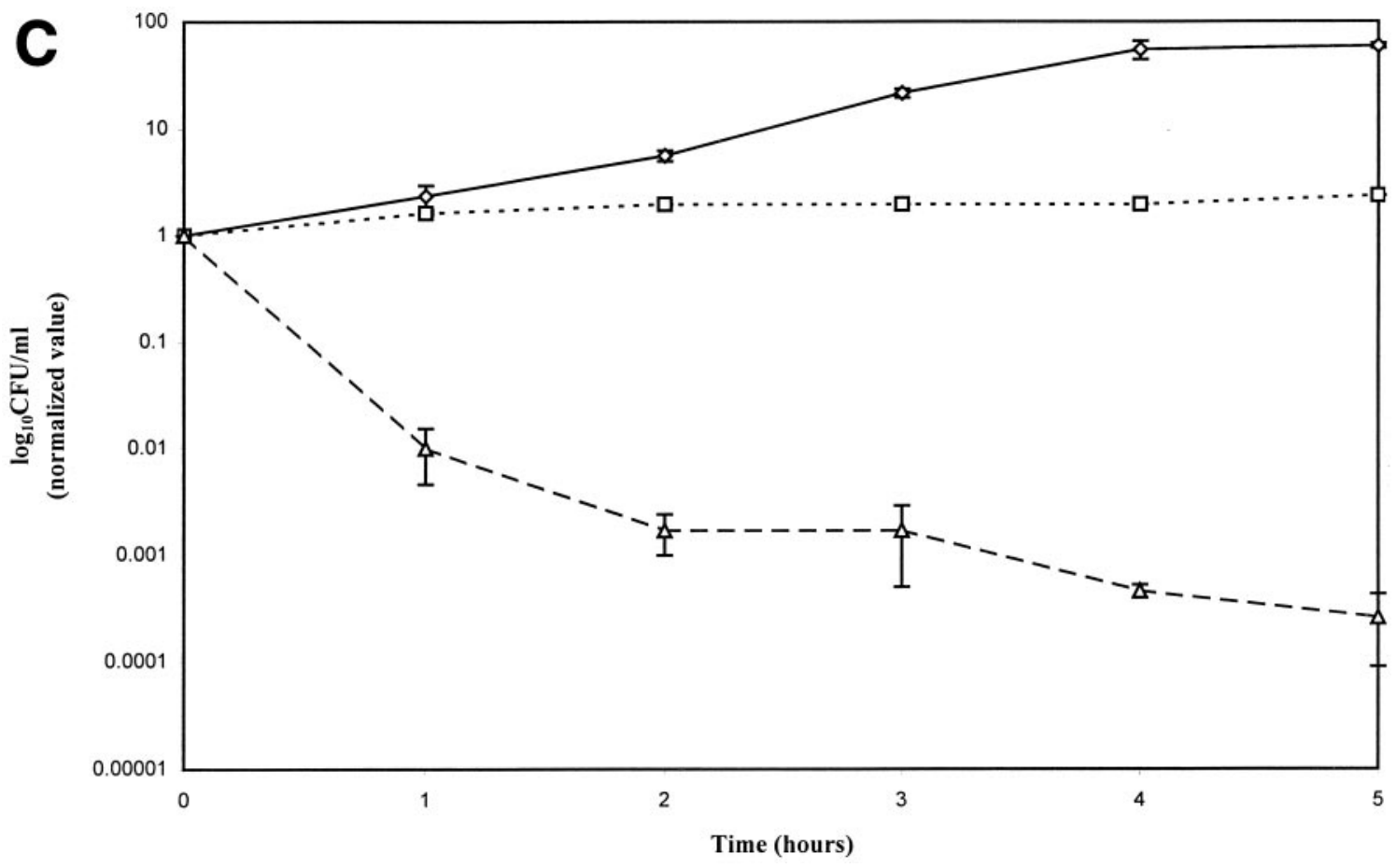

FIG. 1-Continued.

lection of mutants without topoisomerase mutations and without evidence of increased efflux at low selecting concentrations of moxifloxacin has also been reported for $S$. pneumoniae $(10,14)$.

Selection and characterization of serial passage mutants. Serial passage of ISP794 on brain heart infusion agar containing twofold-increasing concentrations of moxifloxacin yielded mutants with up to a 64- to 128 -fold increase in the MIC $(4.0 \mu \mathrm{g} / \mathrm{ml})$. The MICs of moxifloxacin and ciprofloxacin were increased 16- to 32-fold and 8- to 16-fold, respectively, for mutant M8, the most resistant mutant in one series, and 64- to 128-fold and 128- to 256-fold for mutant M11, the most resistant mutant in a second independent series (Table 2). The MICs of nalidixic acid, novobiocin, and ethidium bromide showed at most a twofold change (data not shown). Direct sequencing of the PCR products for the QRDRs of $\operatorname{grl} B A$ revealed a novel mutation (Arg17His) and a commonly encountered mutation (Glu87Lys) in grlA, and sequencing of the QRDRs of gyrBA revealed a common mutation in gyrA (Ser84Leu) (Table 2).

Activities of moxifloxacin and ciprofloxacin against purified

TABLE 3. Frequency of selection of resistant ISP794 mutants

\begin{tabular}{cccccc}
\hline $\begin{array}{c}\text { Selecting } \\
\text { drug } \\
\text { concn } \\
(\mu \mathrm{g} / \mathrm{ml})\end{array}$ & \begin{tabular}{c} 
Factor of MIC \\
\cline { 5 - 5 } floxacin
\end{tabular} & $\begin{array}{c}\text { Moxi- } \\
\text { floxacin }\end{array}$ & Moxifloxacin & Ciprofloxacin \\
\hline 0.064 & 2 & & $4.2 \times 10^{-6}$ & \\
0.125 & 4 & & $4.5 \times 10^{-11}$ & \\
0.25 & 8 & & $<4.5 \times 10^{-11}$ & \\
0.5 & & 2 & & $2.8 \times 10^{-6}-1.5 \times 10^{-5}$ \\
1.0 & & 4 & & $3.0 \times 10^{-8}-6.1 \times 10^{-8}$ \\
2.0 & & 8 & & $2.8 \times 10^{-9}-<4.5 \times 10^{-11}$ \\
\hline
\end{tabular}

topoisomerase IV and gyrase. The cloning of $\operatorname{grl} A, \operatorname{grl} B$, gyr $A$, and gyr $B$ into expression vectors pTrcHis $\mathrm{A},-\mathrm{B}$, or $-\mathrm{C}$, purification to $>90 \%$ homogeneity, and reconstitution of histidine-tagged topoisomerase IV and DNA gyrase from wild-type S. aureus ISP794 and topoisomerase IV from the GrlA mutant (Ser80Phe) as well as the specific activities of the recombinant proteins and the assay conditions for kinetoplast (kDNA) decatenation and DNA supercoiling assays have been reported previously (13). The drug concentrations of moxifloxacin and ciprofloxacin causing 50\% inhibition of kDNA decatenation by topoisomerase IV $\left(\mathrm{IC}_{50}\right)$, estimated visually, were similar or twofold lower for moxifloxacin (1.25 to $2.5 \mu \mathrm{g} / \mathrm{ml}$ and 2.5 to $5.0 \mu \mathrm{g} / \mathrm{ml}$, respectively) (Table 4; Fig. $2 \mathrm{~A})$, and the $\mathrm{IC}_{50}$ for the GrlA mutant increased similarly for both quinolones (up to 100 to $200 \mu \mathrm{g} / \mathrm{ml}$ and $250 \mu \mathrm{g} / \mathrm{ml}$, respectively) (Fig. 2B). The drug concentration causing 50\% inhibition of DNA supercoiling by DNA gyrase $\left(\mathrm{IC}_{50}\right)$ was two- to fourfold lower for moxifloxacin than for ciprofloxacin (2.5 to 5.0 and 10 $\mu \mathrm{g} / \mathrm{ml}$, respectively), suggesting a higher activity of moxifloxacin

TABLE 4. Inhibitory effects of moxifloxacin and ciprofloxacin on wild-type and mutant (GrlA) topoisomerase IV and wild-type gyrase

\begin{tabular}{|c|c|c|c|c|c|c|}
\hline \multirow{2}{*}{ Enzyme } & \multicolumn{4}{|c|}{ Subunit } & \multicolumn{2}{|c|}{$\mathrm{IC}_{50}(\mu \mathrm{g} / \mathrm{ml})$} \\
\hline & GrlA & GrlB & GyrA & GyrB & $\begin{array}{l}\text { Moxi- } \\
\text { floxacin }\end{array}$ & $\begin{array}{l}\text { Cipro- } \\
\text { floxacin }\end{array}$ \\
\hline $\begin{array}{l}\text { Topoisomerase } \\
\text { IV }\end{array}$ & $\begin{array}{l}\text { Wild } \\
\text { type }\end{array}$ & $\begin{array}{l}\text { Wild } \\
\text { type }\end{array}$ & & & $1.25-2.5$ & $2.5-5.0$ \\
\hline $\begin{array}{l}\text { Topoisomerase } \\
\text { IV }\end{array}$ & Phe80 & $\begin{array}{l}\text { Wild } \\
\text { type }\end{array}$ & & & $100-200$ & 250 \\
\hline Gyrase & & & $\begin{array}{l}\text { Wild } \\
\text { type }\end{array}$ & $\begin{array}{l}\text { Wild } \\
\text { type }\end{array}$ & $2.5-5.0$ & 10 \\
\hline
\end{tabular}


A)
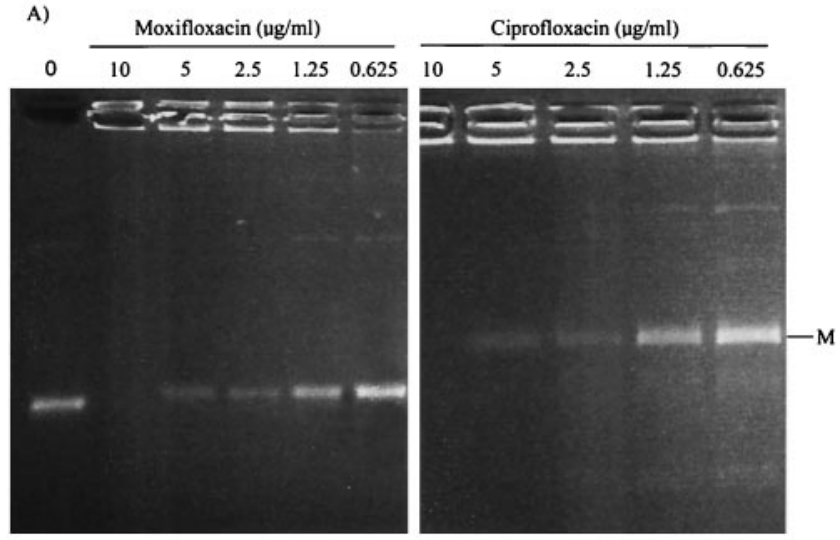

B)

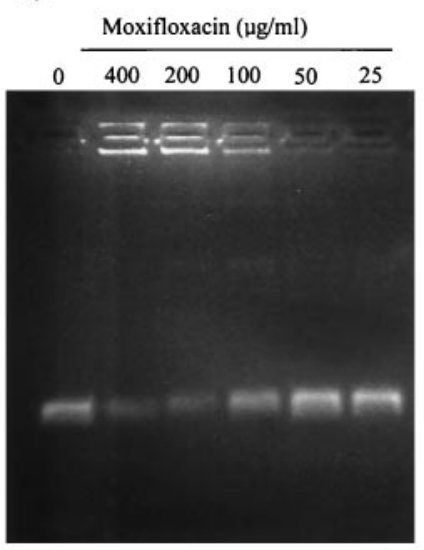

Ciprofloxacin $(\mu \mathrm{g} / \mathrm{ml})$

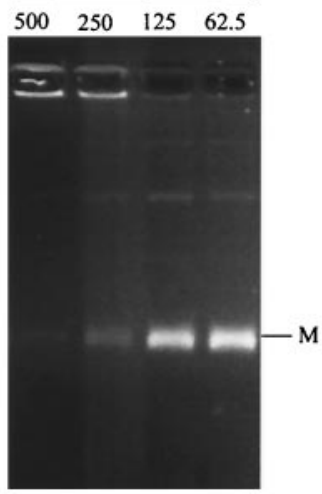

C)

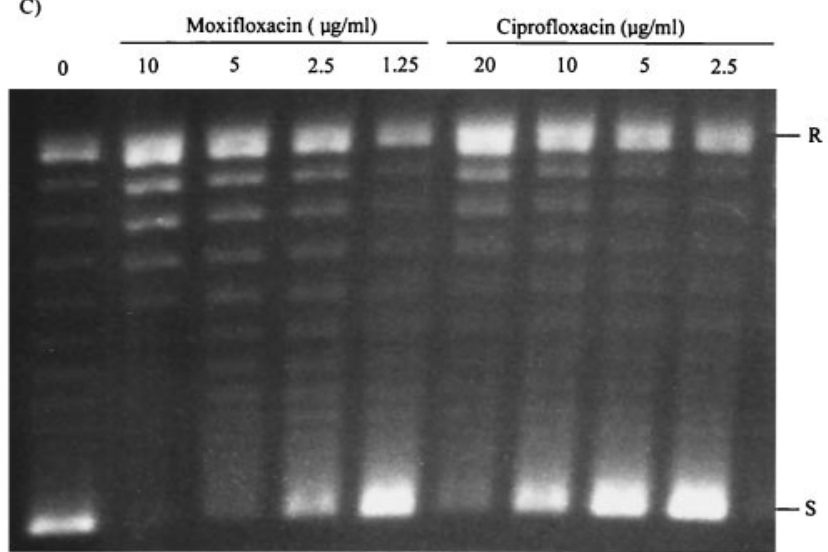

FIG. 2. Activity of moxifloxacin and ciprofloxacin against purified topoisomerase IV and DNA gyrase. (A) Decatenation of kDNA by wild-type GrlA and GrlB in the presence of moxifloxacin and ciprofloxacin. Assays were performed as described in the text. M, minicircles. (B) Decatenation of kDNA by GrlA (Ser80Phe) and wild-type GrlB in the presence of moxifloxacin and ciprofloxacin. (C) DNA supercoiling activity of gyrase in the presence of moxifloxacin and ciprofloxacin. R and S indicate relaxed and supercoiled DNA, respectively.

against gyrase than that of ciprofloxacin (Fig. 2C). Under the assay conditions used in this study, which were different for the two assays and possibly different from the conditions in the bacterium, inhibition of topoisomerase IV and DNA gyrase by moxi- floxacin was similar, if not slightly better, for topoisomerase IV, suggesting similar targeting of both enzymes.

Despite similar inhibition of topoisomerase IV and DNA gyrase by moxifloxacin and ciprofloxacin, moxifloxacin was four- to eightfold more potent against $S$. aureus than ciprofloxacin. This discrepancy might be due to the slightly better targeting of both topoisomerase IV (at most twofold) and DNA gyrase (two- to fourfold) by moxifloxacin in comparison to that of ciprofloxacin, as well as to moxifloxacin not being a good substrate for NorA and possibly other efflux pumps. Moxifloxacin also exerts a greater bactericidal activity against $S$. aureus and its mutants than ciprofloxacin and selects for resistant mutants less frequently. Previously, Takei et al. have also suggested that moxifloxacin is a dual topoisomerase targeting quinolone, based on MIC ratios for topoisomerase IV and DNA gyrase mutants and inhibition of the two target enzymes (26). Our results of in vitro activity of moxifloxacin against reconstituted $S$. aureus topoisomerase IV and DNA gyrase are similar to the data of Takei et al.; however, in our genetically defined mutants, we found a higher increment in the MIC of moxifloxacin in topoisomerase IV mutants than in a gyrase mutant. This finding, together with the initial selection of topoisomerase IV, suggest that moxifloxacin, similar to other 8-methoxyquinolones, preferentially targets topoisomerase IV in vivo in $S$. aureus. Finally, studies with newer quinolones have made localization of target gene mutations to the QRDRs much less consistent, implying more complex interactions among topoisomerases, DNA, and quinolones.

This work was supported by a grant from the U.S. Public Health Service, National Institutes of Health (R01 AI23988 to D.C.H.), and a grant from Bayer Corporation.

\section{REFERENCES}

1. Balfour, J. A., and L. R. Wiseman. 1999. Moxifloxacin. Drugs 57:363-373.

2. Bauernfeind, A. 1997. Comparison of the antibacterial activities of the quinolones BAY 12-8039, gatifloxacin (AM 1155), trovafloxacin, clinafloxacin, levofloxacin and ciprofloxacin. J. Antimicrob. Chemother. 40:639-651.

3. Bisognano, C., P. E. Vaudaux, D. P. Lew, E. Y. W. Ng, and D. C. Hooper. 1997. Increased expression of fibronectin-binding proteins by fluoroquinolone-resistant Staphylococcus aureus exposed to subinhibitory levels of ciprofloxacin. Antimicrob. Agents Chemother. 41:906-913.

4. Dong, Y. Z., C. Xu, X. L. Zhao, J. Domagala, and K. Drlica. 1998. Fluoroquinolone action against mycobacteria: effects of C-8 substituents on growth, survival, and resistance. Antimicrob. Agents Chemother. 42:2978-2984.

5. Dong, Y. Z., X. L. Zhao, J. Domagala, and K. Drlica. 1999. Effect of fluoroquinolone concentration on selection of resistant mutants of Mycobacterium bovis BCG and Staphylococcus aureus. Antimicrob. Agents Chemother. 43:1756-1758.

6. Entenza, J. M., Y. A. Que, J. Vouillamoz, M. P. Glauser, and P. Moreillon. 2001. Efficacies of moxifloxacin, ciprofloxacin, and vancomycin against experimental endocarditis due to methicillin-resistant Staphylococcus aureus expressing various degrees of ciprofloxacin resistance. Antimicrob. Agents Chemother. 45:3076-3083

7. Fournier, B., and D. C. Hooper. 1998. Effects of mutations in GrlA of topoisomerase IV from Staphylococcus aureus on quinolone and coumarin activity. Antimicrob. Agents Chemother. 42:2109-2112.

8. Fournier, B., and D. C. Hooper. 1998. Mutations in topoisomerase IV and DNA gyrase of Staphylococcus aureus: novel pleiotropic effects on quinolone and coumarin activity. Antimicrob. Agents Chemother. 42:121-128.

9. Hoogkamp-Korstanje, J. A. A., and J. Roelofs-Willemse. 2000. Comparative in vitro activity of moxifloxacin against gram-positive clinical isolates. J. Antimicrob. Chemother. 45:31-39.

10. Houssaye, S., L. Gutmann, and E. Varon. 2002. Topoisomerase mutations associated with in vitro selection of resistance to moxifloxacin in Streptococcus pneumoniae. Antimicrob. Agents Chemother. 46:2712-2715.

11. Ince, D., and D. C. Hooper. 2000. Mechanisms and frequency of resistance to premafloxacin in Staphylococcus aureus: novel mutations suggest novel drugtarget interactions. Antimicrob. Agents Chemother. 44:3344-3350.

12. Ince, D., and D. C. Hooper. 2001. Mechanisms and frequency of resistance to 
gatifloxacin in comparison to AM-1121 and ciprofloxacin in Staphylococcus aureus. Antimicrob. Agents Chemother. 45:2755-2764.

13. Ince, D., X. Zhang, L. C. Silver, and D. C. Hooper. 2002. Dual targeting of DNA gyrase and topoisomerase IV. Target interactions of garenoxacin (BMS-284756, T-3811ME), a new desfluoroquinolone. Antimicrob. Agents Chemother. 46:3370-3380.

14. Li, X. Y., X. L. Zhao, and K. Drlica. 2002. Selection of Streptococcus pneumoniae mutants having reduced susceptibility to moxifloxacin and levofloxacin. Antimicrob. Agents Chemother. 46:522-524.

15. Malathum, K., K. V. Singh, and B. E. Murray. 1999. In vitro activity of moxifloxacin, a new 8-methoxyquinolone, against gram-positive bacteria. Diagn. Microbiol. Infect. Dis. 35:127-133.

16. Milatovic, D., F. J. Schmitz, S. Brisse, J. Verhoef, and A. C. Fluit. 2000. In vitro activities of sitafloxacin (DU-6859a) and six other fluoroquinolones against 8,796 clinical bacterial isolates. Antimicrob. Agents Chemother. 44: 1102-1107.

17. Ng, E. Y., M. Trucksis, and D. C. Hooper. 1994. Quinolone resistance mediated by norA: physiologic characterization and relationship to $f l q B$, a quinolone resistance locus on the Staphylococcus aureus chromosome. Antimicrob. Agents Chemother. 38:1345-1355.

18. Ng, E. Y., M. Trucksis, and D. C. Hooper. 1996. Quinolone resistance mutations in topoisomerase IV: relationship of the flqA locus and genetic evidence that topoisomerase IV is the primary target and DNA gyrase the secondary target of fluoroquinolones in Staphylococcus aureus. Antimicrob. Agents Chemother. 40:1881-1888.

19. Pestova, E., J. J. Millichap, G. A. Noskin, and L. R. Peterson. 2000. Intracellular targets of moxifloxacin: a comparison with other fluoroquinolones. J. Antimicrob. Chemother. 45:583-590.

20. Piddock, L. J., and Y. F. Jin. 1999. Antimicrobial activity and accumulation of moxifloxacin in quinolone-susceptible bacteria. J. Antimicrob. Chemother. 43:39-42.

21. Pong, A., K. S. Thomson, E. S. Moland, S. A. Chartrand, and C. C. Sanders 1999. Activity of moxifloxacin against pathogens with decreased susceptibility to ciprofloxacin. J. Antimicrob. Chemother. 44:621-627.

22. Schedletzky, H., B. Wiedemann, and P. Heisig. 1999. The effect of moxifloxacin on its target topoisomerases from Escherichia coli and Staphylococcus aureus. J. Antimicrob. Chemother. 43:31-37.
23. Sindelar, G., X. Zhao, A. Liew, Y. Dong, T. Lu, J. Zhou, J. Domagala, and K. Drlica. 2000. Mutant prevention concentration as a measure of fluoroquinolone potency against mycobacteria. Antimicrob. Agents Chemother. 44: 3337-3343.

24. Stahl, M. L., and P. A. Pattee. 1983. Confirmation of protoplast fusionderived linkages in Staphylococcus aureus by transformation with protoplast DNA. J. Bacteriol. 154:406-412.

25. Stass, H., A. Dalhoff, D. Kubitza, and U. Schühly. 1998. Pharmacokinetics, safety, and tolerability of ascending single doses of moxifloxacin, a new 8-methoxy quinolone, administered to healthy subjects. Antimicrob. Agents Chemother. 42:2060-2065.

26. Takei, M., H. Fukuda, R. Kishii, and M. Hosaka. 2001. Target preference of 15 quinolones against Staphylococcus aureus, based on antibacterial activities and target inhibition. Antimicrob. Agents Chemother. 45:3544-3547.

27. Takenouchi, T., F. Tabata, Y. Iwata, H. Hanzawa, M. Sugawara, and S. Ohya. 1996. Hydrophilicity of quinolones is not an exclusive factor for decreased activity in efflux-mediated resistant mutants of Staphylococcus aureus. Antimicrob. Agents Chemother. 40:1835-1842.

28. Trucksis, M., J. S. Wolfson, and D. C. Hooper. 1991. A novel locus conferring fluoroquinolone resistance in Staphylococcus aureus. J. Bacteriol. 173: 5854-5860.

29. Varon, E., C. Janoir, M. D. Kitzis, and L. Gutmann. 1999. ParC and GyrA may be interchangeable initial targets of some fluoroquinolones in Streptococcus pneumoniae. Antimicrob. Agents Chemother. 43:302-306.

30. Von Eiff, C., and G. Peters. 1999. Comparative in-vitro activities of moxifloxacin, trovafloxacin, quinupristin/dalfopristin and linezolid against staphylococci. J. Antimicrob. Chemother. 43:569-573.

31. Woodcock, J. M., J. M. Andrews, F. J. Boswell, N. P. Brenwald, and R. Wise. 1997. In vitro activity of BAY 12-8039, a new fluoroquinolone. Antimicrob. Agents Chemother. 41:101-106.

32. Yague, G., J. E. Morris, X. S. Pan, K. A. Gould, and L. M. Fisher. 2002. Cleavable-complex formation by wild-type and quinolone-resistant Streptococcus pneumoniae type II topoisomerases mediated by gemifloxacin and other fluoroquinolones. Antimicrob. Agents Chemother. 46:413-419.

33. Zhao, X. L., J. Y. Wang, C. Xu, Y. Z. Dong, J. F. Zhou, J. Domagala, and K. Drlica. 1998. Killing of Staphylococcus aureus by C-8-methoxy fluoroquinolones. Antimicrob. Agents Chemother. 42:956-958. 